\title{
Laboratory Verification of a BRCA1 and BRCA2 Massively Parallel Sequencing Assay from Wet Bench to Bioinformatics for Germline DNA Analysis
}

\author{
Kok-Siong Poon ${ }^{1}$ Lily Chiu ${ }^{1}$ Karen Mei-Ling $\operatorname{Tan}^{1}$ \\ ${ }^{1}$ Department of Laboratory Medicine, National University Hospital \\ Singapore, Singapore \\ Global Med Genet 2021;8:62-68.
}

Address for correspondence Kok-Siong Poon, MSc, Department of Laboratory Medicine, National University Hospital, 5 Lower Kent Ridge Road, 119074 Singapore (e-mail: kok_siong_poon@nuhs.edu.sg).

\begin{abstract}
Introduction A robust genetic test for BRCA1 and BRCA2 genes is necessary for the diagnosis, prognosis, and treatment of patients with hereditary breast and ovarian cancer. We evaluated a commercial amplicon-based massively parallel sequencing (MPS) assay, BRCA MASTR Plus on the MiSeq platform, for germline BRCA genetic testing.

Methods This study was performed on 31 DNA from cell lines and proficiency testing samples to establish the accuracy of the assay. A reference cell line DNA, NA12878 was used to determine the reproducibility of the assay. Discordant MPS result was resolved orthogonally by the current gold-standard Sanger sequencing method.

Results The analytical accuracy, sensitivity, and specificity for variant detection were 93.55, 92.86, and $100.00 \%$, respectively. Both sequencing depth and variant allele

Keywords

- BRCA1

- BRCA2

- massively parallel sequencing

- NGS

- Multiplicom frequencies were highly reproducible by comparing the NA12878 DNA tested in three separate runs. The single discordant result, later confirmed by Sanger sequencing was due to the inability of the MASTR Reporter software to identify a 40-bp deletion in BRCA1.

Conclusion The BRCA MASTR Plus assay on the MiSeq platform is accurate and reproducible for germline $B R C A$ genetic testing, making it suitable for use in a clinical diagnostic laboratory. However, Sanger sequencing may still serve as a confirmatory method to improve diagnostic capability of the MPS assay.
\end{abstract}

\section{Introduction}

Hereditary breast and ovarian cancer (HBOC) is an autosomal dominant cancer syndrome frequently caused by germline pathogenic variants in the two DNA repair genes, BRCA1 and $B R C A 2$. Due to high penetrance, approximately $50 \%$ of women with $B R C A$ pathogenic variants will be diagnosed with breast cancer by age of 70 years. ${ }^{1}$ For ovarian cancer, these estimates were 40 and $18 \%$ of women with mutant BRCA1 and BRCA2 genes, respectively. ${ }^{1}$ Several poly ADP ribose polymerases (PARP) inhibitors have been approved for therapy in patients with HBOC syndrome with germline BRCA pathogenic variants. ${ }^{2}$ In patients with $>30 \%$ variant allele published online March 16, 2021
DOI https://doi.org/ 10.1055/s-0041-1726338. ISSN 2699-9404.

\section{(c) 2021. The Author(s).}

This is an open access article published by Thieme under the terms of the Creative Commons Attribution License, permitting unrestricted use, distribution, and reproduction so long as the original work is properly cited. (https://creativecommons.org/licenses/by/4.0/)

Georg Thieme Verlag KG, Rüdigerstraße 14, 70469 Stuttgart, Germany 
frequency (VAF) of pathogenic BRCA variants from tumor profiling, genetic testing of germline variant is recommended. ${ }^{3}$ Genetic testing of the BRCA genes plays a vital role to allow identification of carriers of pathogenic variants and increased screening for early detection of breast and ovarian cancers in these individuals. A robust laboratory assay is crucial to enable genetic testing of BRCA1 and BRCA2 genes on which risk assessment, patient management, and therapeutic assignment in HBOC patients rely.

The BRCA1 (OMIM: 113705) and BRCA2 (OMIM: 600185) genes, located at 17q21.31 and 13q13.1, are large genes with 24 and 27 exons encoding 1,863 and 3,418 amino acids, respectively. They are tumor-suppressor genes in which lossof-function variants are associated with increased risk of HBOC syndrome. A wide spectrum of pathogenic variants is detectable throughout the coding and splice site regions of the BRCA genes. These genetic alterations are heterogeneous, including single nucleotide variants (SNVs), small insertions and deletions (indels) affecting a short stretch of nucleotides, large indels at exonic level and copy number variants (CNV). With the advent of massively parallel sequencing (MPS), the laboratory accessibility to sequencing the two large $B R C A$ genes is improved. A recent international survey ${ }^{4}$ revealed that $93 \%$ of the laboratories utilize MPS platforms for sequencing the $B R C A$ genes. In this study, we evaluated a commercial amplicon-based MPS assay, BRCA MASTR Plus (Multiplicom, Niel, Belgium) on the MiSeq platform (Illumina; San Diego, California, United States), for germline $B R C A 1$ and BRCA2 genetic testing.

\section{Methods}

\section{DNA Samples}

Samples tested in this study were cell line DNA from Coriell Institute for Medical Research $(n=10)$, namely, NA13714, NA14091, NA14624, NA14639, NA14788, NA14805, NA14623, NA14622, NA14170, and NA12878. Notably, NA12878 is a reference cell line characterized by the Genome in a Bottle (GIAB) Consortium hosted by National Institute of Standards and Technology (NIST). ${ }^{5}$ Twenty-one DNA samples accrued from College of American Pathologists/ American College of Medical Genetics (CAP/ACMG) BRCA1/2 Sequencing External Quality Assurance (EQA) Program were also tested in this study.

\section{BRCA MASTR Plus Assay Library Preparation and \\ Sequencing}

Concentrations of DNA samples were measured using NanoDrop 2000 (Thermo Fisher Scientific, Waltham, Massachusetts, United States). Working DNA samples were diluted with nuclease-free water (Invitrogen, Waltham, Massachusetts, United States) to a concentration of $10 \mathrm{ng} / \mu \mathrm{L}$. Five $\mu \mathrm{L}$ of diluted DNA was subject to four multiplex polymerase chain reactions (PCRs) with reagents supplied in BRCA MASTR Plus kit (Multiplicom) according to manufacturer's instructions. Five $\mu \mathrm{L}$ of PCR products from the multiplex PCR reactions were subject to electrophoresis using $2 \%(\mathrm{w} / \mathrm{v})$ agarose gel in Tris-Borate-EDTA (TBE) buffer at $100 \mathrm{~V}$ for 30 minutes to verify the presence of PCR products with sizes exceeding 150 base pairs. Equal volumes of multiplex PCR products were pooled and purified with AMPure XP beads (Agencourt; Beverly, Massachusetts, United States). Universal PCR was performed on purified pooled PCR products with MID p7 and p5 adaptor and primer mixes supplied in drMID for Illumina NGS systems kit (Multiplicom). Five $\mu \mathrm{L}$ of the universal PCR products were subject to electrophoresis using $2 \%(\mathrm{w} / \mathrm{v})$ agarose gel in TBE buffer at $100 \mathrm{~V}$ for 30 minutes to verify the presence of PCR products with sizes exceeding 200 base pairs. The universal PCR products purified with AMPure XP beads were diluted to $4 \mathrm{nM}$ in TE buffer (Thermo Fisher Scientific) and pooled into a single library. The library was diluted to $12 \mathrm{pM}$ and denatured using $0.2 \mathrm{~N}$ sodium hydroxide $(\mathrm{NaOH})$. MPS was performed on the MiSeq system (Illumina) using MiSeq Reagent Micro Kit v2 (300 cycles).

\section{Bioinformatics}

The demultiplexed FASTQ sequence files were uploaded to MASTR Reporter v1.2.1, proprietary web-based software by Multiplicom. The application of BRCA MASTR Plus Dx Germline was selected to analyze the sequencing data in this study. For variant analysis, the minimum coverage depth and allele frequency were specified at $40 \times$ and $20 \%$, respectively. Variants were classified according to the 2015 ACMG/AMP guidelines. $^{6}$

\section{Sanger Confirmation}

A set of primers was designed using Primer3 software to amplify exon 11 of the BRCA1 gene with expected PCR product size of 430 base pairs (bp). The forward and reverse primers were 5'cagaaactgccatgctcaga3' and 5'tgaggggacgctcttgtatt3', respectively. PCR was performed using HotStarTaq Plus Master Mix Kit (QIAGEN; Hilden, Germany) on 50 ng of DNA input. PCR products were purified with GeneAll Expin Kit (GeneAll Biotechnology, Seoul, Korea) and subject to cycle sequencing reaction using the same forward and reverse PCR primers with BigDye Terminator v3.1 Cycle Sequencing kit (Thermo Fisher Scientific). Sanger sequencing was performed on cycle sequencing products purified with DyeEx 2.0 Spin kit (QIAGEN) on ABI 3130 Genetic Analyzer (Thermo Fisher Scientific). Sequence analysis was performed using ATF software (Conexio Genomics, Fremantle, Australia).

\section{Statistical Analysis}

Accuracy was calculated as the number of true positives and true negatives divided by the sum of true positives, true negatives, false positives, and false negatives using an online statistical software, MEDCALC.

\section{Results}

Sequencing Depth and Reproducibility of the Assay With a pooling strategy with 10 samples per library, the minimum sequencing depth was above $400 \times$ and $350 \times$ for BRCA1 (69 amplicons) and BRCA2 (112 amplicons), 


\section{Interrun reproducibility of VAF for BRCA1 variants}

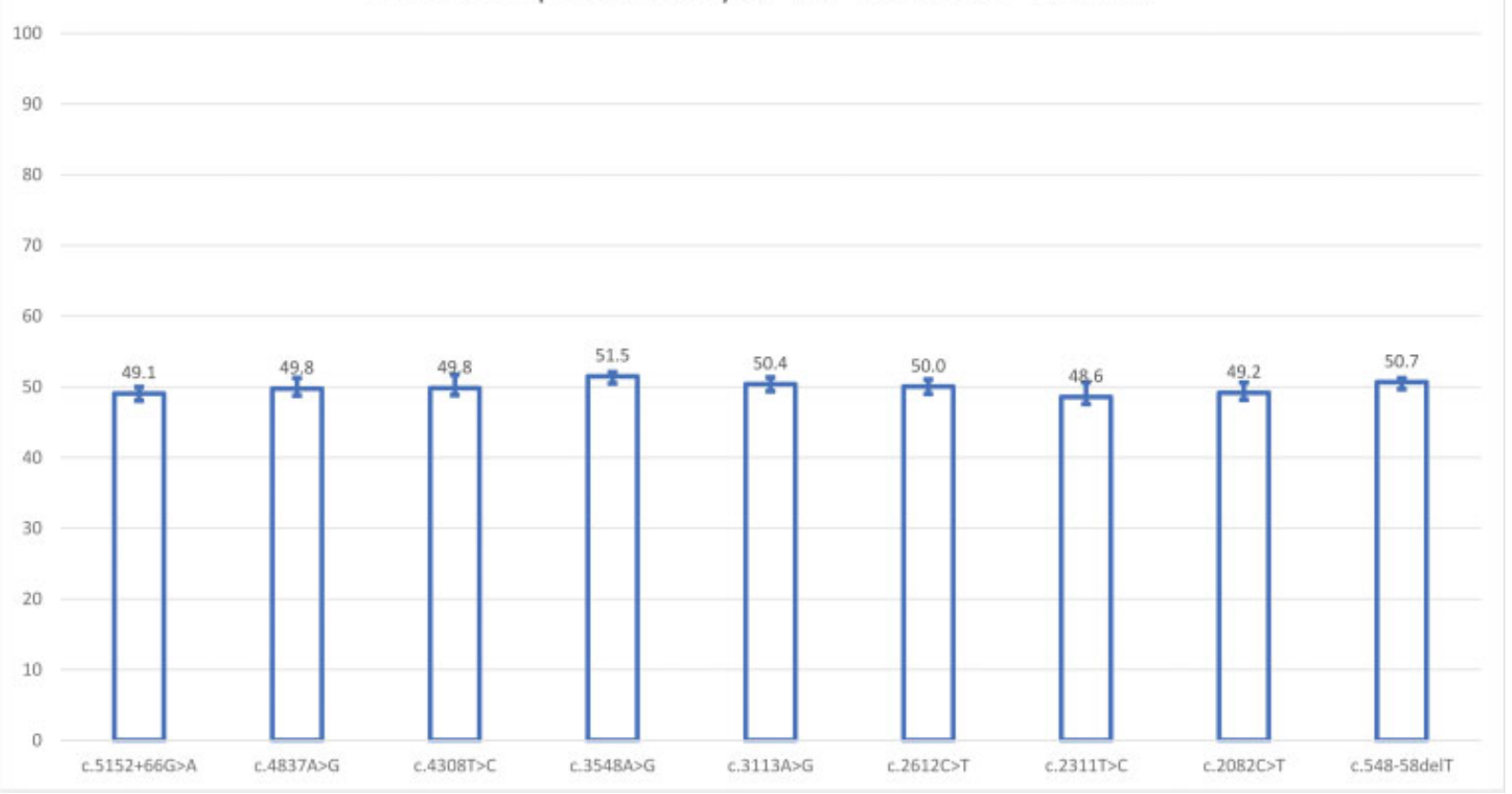

Fig. 3 Interrun reproducibility of variant allele frequency for $B R C A 1$ variants. Bars represent mean and error bars represent standard deviation of three separate runs.

(-Table 1). From these samples, eight heterozygous frameshift variants, three heterozygous stop-gain variants, and three heterozygous SNVs were correctly identified in the BRCA genes. Notably, a 40-bp deletion variant in the BRCA1 gene was not conclusively reported by the MASTR Reporter; however, it was flagged as a long event (-Fig. 5). Sanger sequencing further confirmed the long event as c.1175_1214del40 which was the expected variant according to CAP (-Fig. 6). One EQA DNA sample which did not have any variants was correctly identified as being negative for $B R C A$ variants. Altogether, for a total of 31 DNA samples, covering a range of different variants including SNVs,

\section{Interrun reproducibility of VAF for $B R C A 2$ variants}

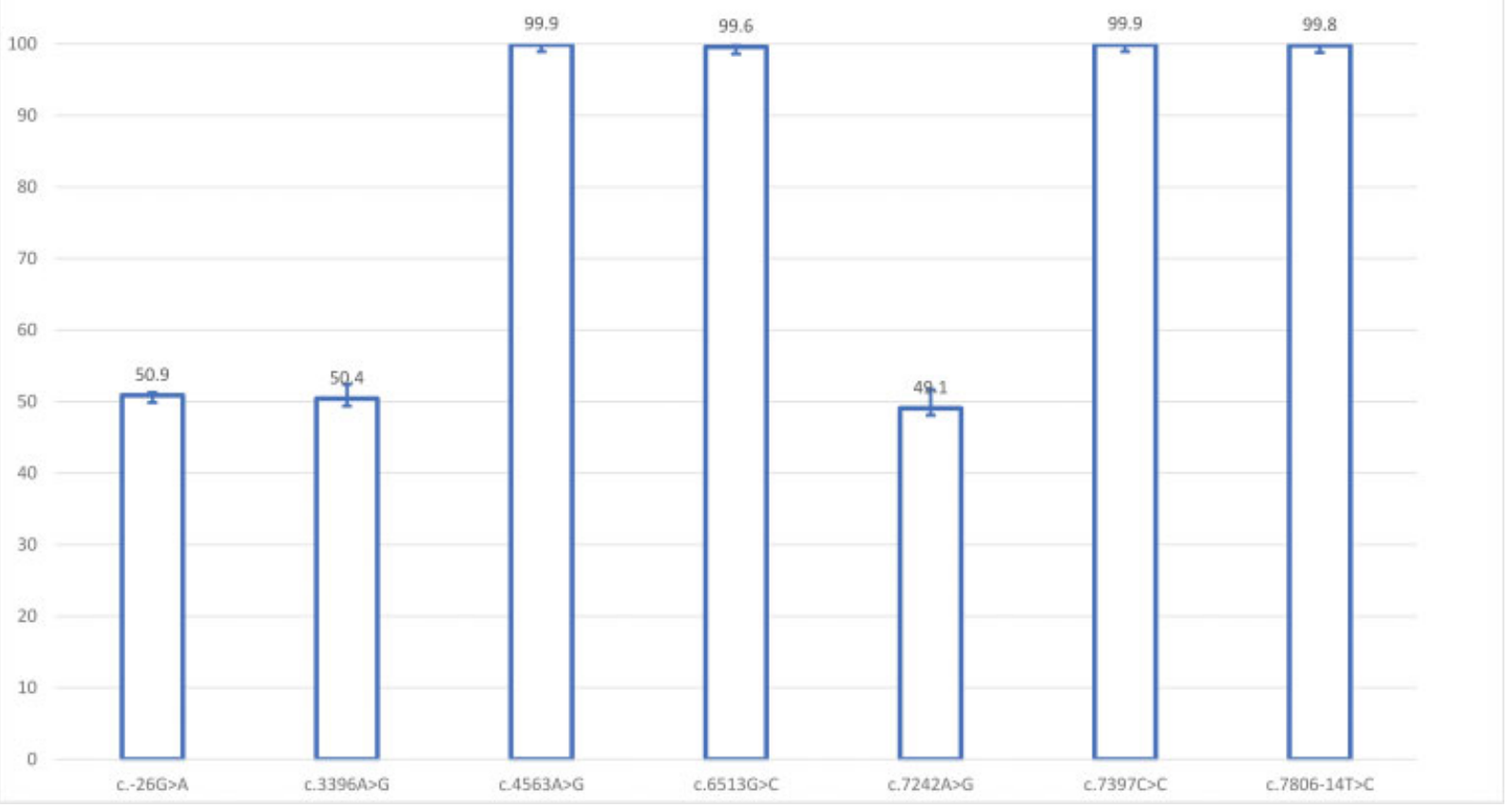

Fig. 4 Interrun reproducibility of variant allele frequency for BRCA2 variants. Bars represent mean and error bars represent standard deviation of three separate runs. 


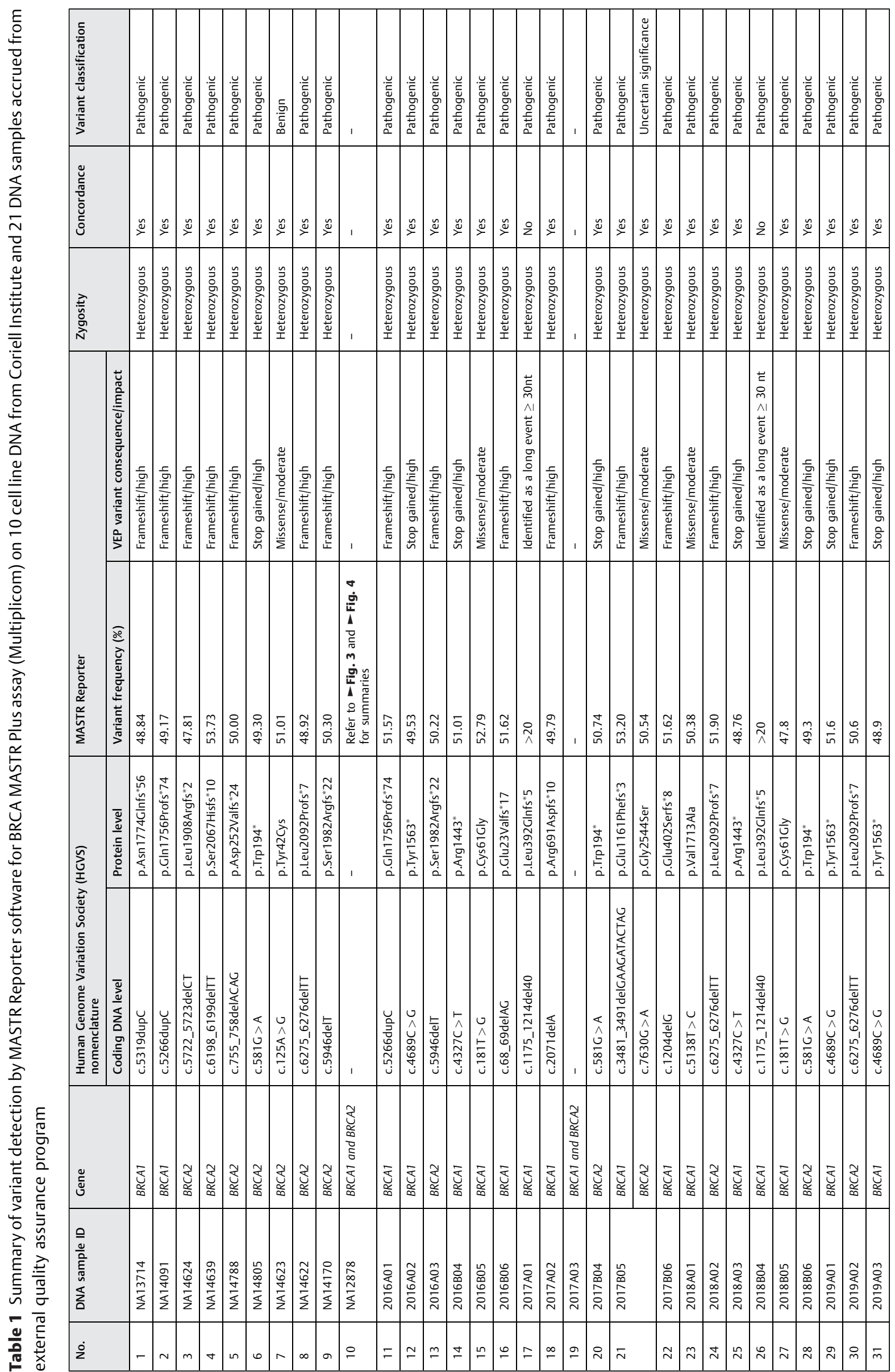




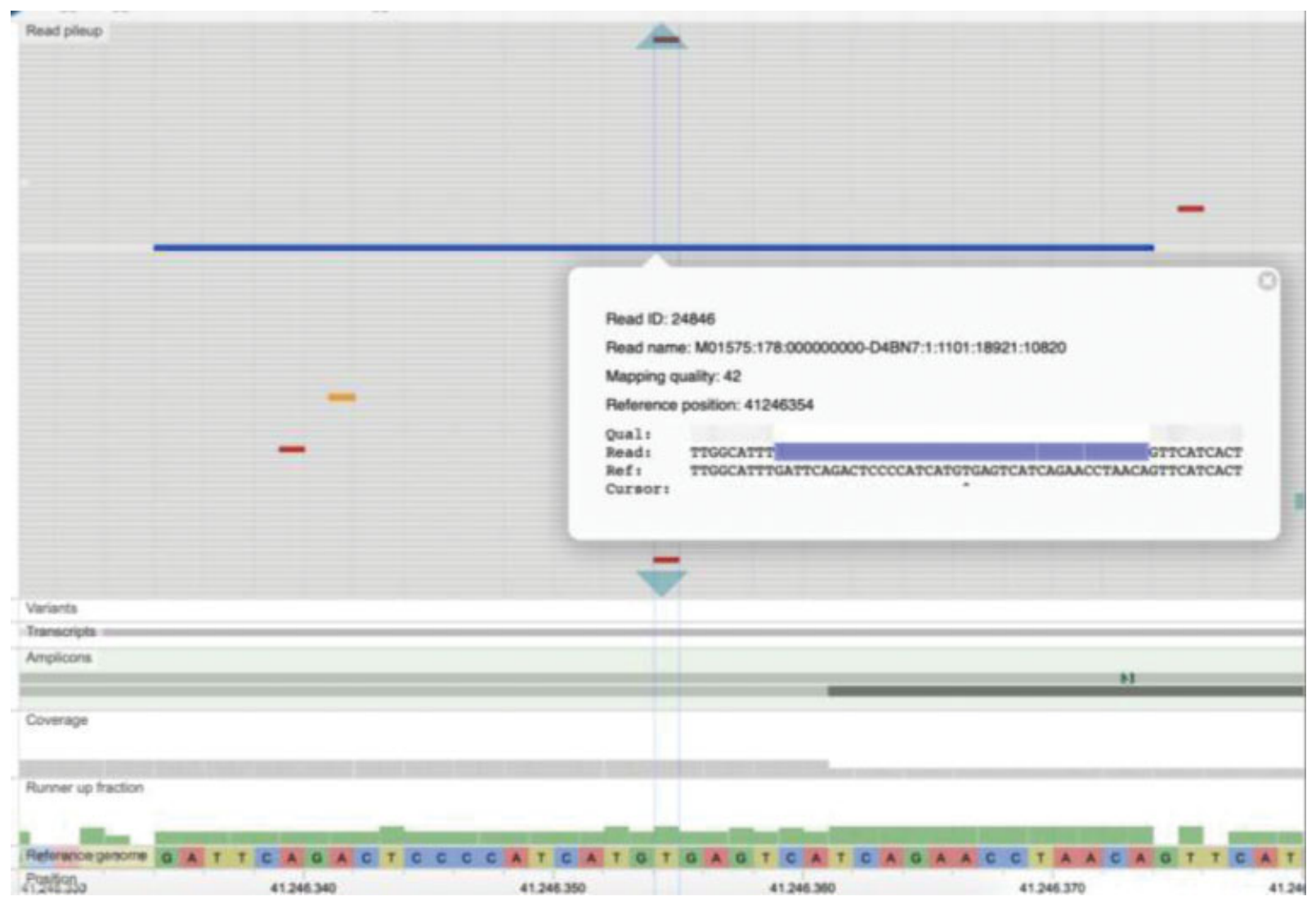

Fig. 5 A long event with 40-bp deletion was equivocally detected on one of the BRCA1 exon 11 amplicons of the BRCA MASTR Plus MPS assay. Mapping of reads to the BRCA1 genomic region was visualized by read pileup in MASTR Reporter. The read with deleted region was abbreviated in blue color. The details including read ID, read name, mapping quality, reference position and nucleotide sequences were shown in the browser. The deletion was later confirmed by PCR and Sanger sequencing (illustrated in - Fig. 6) to be c.1175_1214del40, which was the expected variant according to CAP. CAP, College of American Pathologists; MPS, massively parallel sequencing; PCR, polymerase chain reaction.
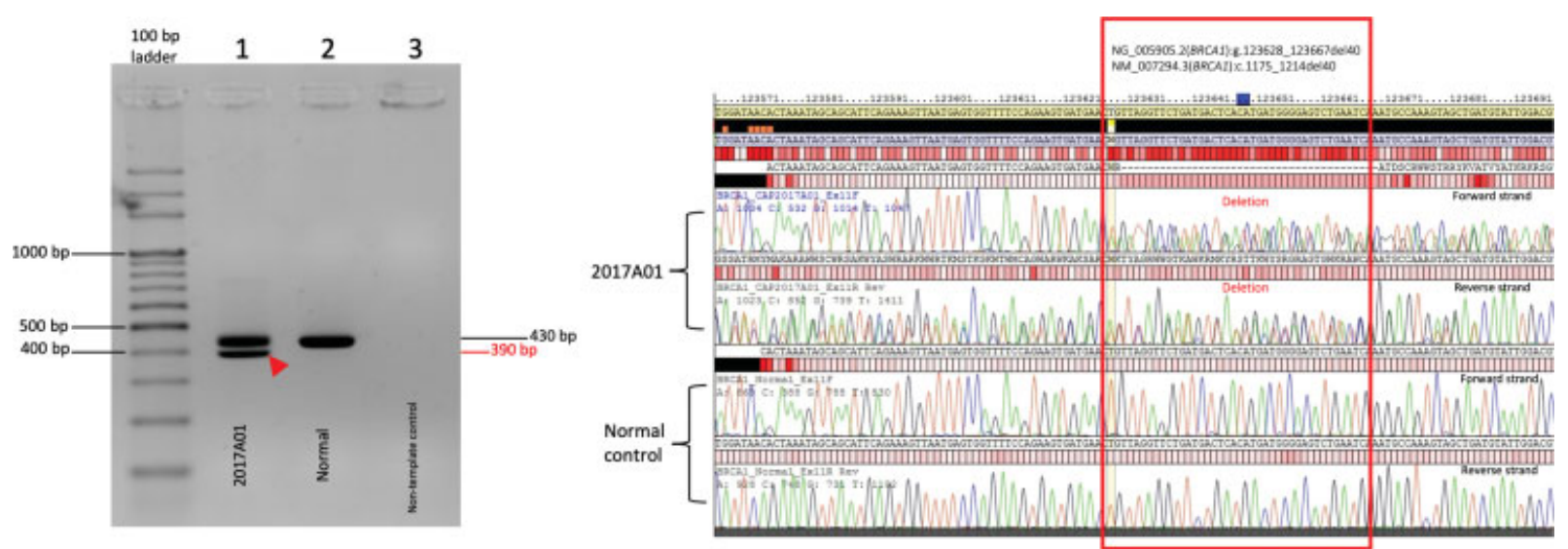

Fig. 6 Confirmation of an equivocal long event detected by MASTR Reporter software using PCR-Sanger sequencing. The BRCA1 genomic region of interest was PCR amplified (left) and followed by Sanger sequencing of the PCR products. The deletion event, c.1175_1214del40 was verified by alignment to the BRCA1 genomic RefSeq NG_005905.2 (right). PCR, polymerase chain reaction.

deletions, and duplications in the BRCA genes, the analytical accuracy for variant detection was $93.55 \%$ (95\% confidence interval [CI]: 78.58-99.21\%). The analytical sensitivity and specificity were $92.86 \%$ (95\% CI: $76.50-99.12 \%$ ) and $100.00 \%$ (95\% CI: 29.24-100.00\%), respectively.

\section{Discussion}

Here we demonstrate the use of well-characterized cell line DNA and blinded proficiency testing samples for evaluation of a commercial MPS assay for the entire coding regions of 
the BRCA1 and BRCA2 genes in germline samples. In the absence of clinical samples, for a clinical laboratory starting a new assay, EQA samples and cell line samples are good resources for evaluating analytical accuracy and precision. Using these samples, we demonstrated the accurate identification of 10 different frameshift variants, 3 different stop gain variants, and 3 different SNVs in the BRCA1 and BRCA2 genes.

All samples showed concordance with the expected variants except for two EQA samples which harbor the same 40bp deletion, c.1175_1214del40, in the BRCA1 gene. First reported in $1994,{ }^{7,8}$ this deletion is not an uncommon pathogenic variant in HBOC patients (ClinVar, accessed on January 10, 2021). The inability to determine insertions and deletions spanning more than 30 -bp is a declared limitation of the assay. Hence the laboratory may need to supplement the MPS assay with Sanger sequencing or use an alternative bioinformatics pipeline to analyze the sequencing data to confirm the exact deletion or insertion.

\section{Conclusion}

In conclusion, we have shown high reproducibility and accuracy of the BRCA MASTR Plus assay on the MiSeq platform. The simple bench workflow in combination with rapid automated data analysis by the MASTR Reporter software make it suitable for use for germline BRCA1 and $B R C A 2$ genetic testing in a clinical diagnostic laboratory. However, Sanger sequencing may still serve as a confirmatory method to improve diagnostic capability of the MPS assay.

\section{Funding}

None.
Conflict of Interests

None declared.

Acknowledgment

The authors are grateful to Dr. Chee Yang Lee and Rosanne Siow from Agilent for their expert advice. We also thank colleagues from the Molecular Diagnosis Centre, NUH, for excellent technical assistance.

\section{References}

1 Chen S, Parmigiani G. Meta-analysis of BRCA1 and BRCA2 penetrance. J Clin Oncol 2007;25(11):1329-1333

2 Faraoni I, Graziani G. Role of BRCA mutations in cancer treatment with poly(ADP-ribose) polymerase (PARP) inhibitors. Cancers (Basel) 2018;10(12):487

3 González-Santiago S, Ramón Y Cajal T, Aguirre E, et al; SEOM Hereditary Cancer Working Group. SEOM clinical guidelines in hereditary breast and ovarian cancer (2019). Clin Transl Oncol 2020;22(02):193-200

4 Toland AE, Forman A, Couch FJ, et al; BIC Steering Committee. Clinical testing of BRCA1 and BRCA2: a worldwide snapshot of technological practices. NPJ Genom Med 2018;3:7

5 Zook JM, Catoe D, McDaniel J, et al. Extensive sequencing of seven human genomes to characterize benchmark reference materials. Sci Data 2016;3:160025

6 Richards S, Aziz N, Bale S, et al; ACMG Laboratory Quality Assurance Committee. Standards and guidelines for the interpretation of sequence variants: a joint consensus recommendation of the American College of Medical Genetics and Genomics and the Association for Molecular Pathology. Genet Med 2015;17(05): 405-424

7 Castilla LH, Couch FJ, Erdos MR, et al. Mutations in the BRCA1 gene in families with early-onset breast and ovarian cancer. Nat Genet 1994;8(04):387-391

8 Simard J, Tonin P, Durocher F, et al. Common origins of BRCA1 mutations in Canadian breast and ovarian cancer families. Nat Genet 1994;8(04):392-398 